\title{
PLANE-WAVE ANALYSIS OF A HYPERBOLIC SYSTEM OF EQUATIONS WITH RELAXATION IN $\mathbb{R}^{d *}$
}

\author{
MAARTEN V. DE HOOP ${ }^{\dagger}$, JIAN-GUO LIU‡, PETER A. MARKOWICH§, AND NAIL S. \\ USSEMBAYEV
}

\begin{abstract}
We consider a multi-dimensional scalar wave equation with memory corresponding to the viscoelastic material described by a generalized Zener model. We deduce that this relaxation system is an example of a non-strictly hyperbolic system satisfying Majda's block structure condition. Wellposedness of the associated Cauchy problem is established by showing that the symbol of the spatial derivatives is uniformly diagonalizable with real eigenvalues. A long-time stability result is obtained by plane-wave analysis when the memory term allows for dissipation of energy.
\end{abstract}

Key words. characteristic fields of constant multiplicity; eigenvalues; viscoelasticity; memory effect; Zener model; stability; energy methods

AMS subject classifications. 35B35; 35L40; 74D05

\section{Introduction}

The theory of viscoelasticity describes materials exhibiting a combination of both elastic solid (deformation eventually disappears when the load is removed) and viscous (Newtonian) fluid characteristics. Wave propagation in viscoelastic unbounded or semibounded media is a relevant idealization in some important real-world problems arising in different fields: geophysics, applied mechanics, material science, acoustics etc.

Viscoelastic materials are modeled by constitutive laws relating the stress to the history of the strain and entering the equation of motion in the form of a convolution integral in time. The resulting integro-differential equation can be written as a system of partial differential equations with a relaxation term and described in Fourier space as an exponential evolution operator acting on a vector representing the initial conditions. The system is hyperbolic when the matrix appearing in the evolution operator is diagonalizable with real eigenvalues and its eigenspace is complete. If, in addition, all eigenvalues are distinct, the system is said to be strictly hyperbolic. One of the important motivations to study strictly hyperbolic systems is that they are invulnerable to perturbations by lower-order terms. Unfortunately, many interesting examples of hyperbolic systems describing various physical phenomena are not strictly hyperbolic and it is not known in general whether such systems remain hyperbolic under perturbations by lower-order terms.

Majda and Osher [12] proved that the strict hyperbolicity assumption used in the construction of Kreiss' symmetrizer could be replaced by a weaker assumption called

*M.V.d.H. gratefully acknowledges support from the Simons Foundation under the MATH + X program, the National Science Foundation under grant DMS-1559587, and the corporate members of the Geo-Mathematical Group at Rice University. J.-G.L. is supported by the National Science Foundation under grant DMS-1514826 and KI-Net RNMS11-07444.

${ }^{\dagger}$ Department of Computational and Applied Mathematics, Rice University, Houston, TX 77005, USA (mdehoop@rice.edu).

$\ddagger$ Department of Mathematics and Department of Physics, Duke University, Durham, NC 27708, USA (jliu@math.duke.edu).

$\S$ Applied Mathematics and Computer Science Program, CEMSE Division, King Abdullah University of Science and Technology, Thuwal 23955-6900, Saudi Arabia and Faculty of Mathematics, University of Vienna, Vienna A-1090, Austria (peter.markowich@kaust.edu.sa).

ฯ Applied Mathematics and Computer Science Program, CEMSE Division, King Abdullah University of Science and Technology Thuwal, 23955-6900, Saudi Arabia (nail.ussembayev@kaust.edu.sa). Corresponding author: Nail S. Ussembayev 
the "block structure condition" which is satisfied by several non-strictly hyperbolic systems including Maxwell's equations of electrodynamics, the linearized shallow water equations and the Euler equations of gas dynamics. However, each system of interest required a separate verification of this property due to the lack of a universal criterion. This was the state of affairs until Métivier [14] extended Majda's work establishing the block structure condition for a class of hyperbolic systems with characteristic fields of constant multiplicity. It is common to refer to such systems simply as "constantly hyperbolic," to wit

DeFinition 1.1. The operator

$$
L=\partial_{t}+\sum_{j=1}^{d} A_{j}(x, t) \partial_{x_{j}}
$$

with $A_{j}, B: \mathbb{R}^{d} \times(0, T) \rightarrow \mathbb{M}^{N \times N}(\mathbb{R})$ is called constantly hyperbolic if there exist an integer $m \geq 1$, natural numbers $l_{1}, \ldots, l_{m}$ and real valued functions $\lambda_{1}, \ldots, \lambda_{m}$ analytic away from the origin such that for any $\xi \in \mathbb{S}^{d-1}$ it holds that

$$
\operatorname{det}\left(\lambda I_{N}+\sum_{j=1}^{d} \xi_{j} A_{j}\right)=\prod_{i=1}^{m}\left(\lambda+\lambda_{i}(\xi)\right)^{l_{i}}, \quad l_{1}+\ldots+l_{m}=N
$$

where all the eigenvalues $\lambda_{i}(\xi)$ of the symbol $A(\xi)=\sum_{j=1}^{d} \xi_{j} A_{j}$ are real, semi-simple and satisfy $\lambda_{1}(\xi)<\ldots<\lambda_{m}(\xi)$.

Let us reiterate: if the eigenvalues are semi-simple instead of being simple as in the case of strict hyperbolicity, and their multiplicities remain constant as $\left(\xi_{1}, \ldots, \xi_{d}\right) \in$ $\mathbb{R}^{d} \backslash\{0\}$ varies, then the corresponding system is called constantly hyperbolic. The notion of constant hyperbolicity is a slight generalization of the concept of strict hyperbolicity where the analysis is technically simpler and had allowed more extensive studies in the past. In Sec. 3 we demonstrate the hyperbolicity of our relaxation system by proving that $A(\xi)$ is diagonalizable with real eigenvalues and verify that the diagonalization is well-conditioned on $\mathbb{S}^{d-1}$. More straightforwardly, hyperbolicity can be shown by appealing to the general structure of the eigenvalues of the system since constant hyperbolicity implies hyperbolicity (see Remark 3.1).

In a bounded domain, existence and uniqueness of solutions can be established using the treatment of Lions and Magenes [10] under minimal assumptions on the regularity of the coefficient functions. A classical analysis regarding equations of the type (2.4) is attributed to Dafermos [5]. Here, the domain can be the whole space, but the requirements on the initial conditions exclude plane waves. Blazek et al. [3] proved the same result for systems of equations. Kim [9] obtained existence and uniqueness of solutions using Friedrichs mollifier techniques assuming that the coefficient functions are smooth in space and time while allowing plane-wave initial conditions. Kim's analysis also motivates the development of a microlocal analogue of the correspondence principle [16] in a parametrix construction starting from plane-wave initial values.

Following Bécache et al. [1] we rewrite the system with relaxation based on a generalized Zener solid in first-order partial-differential form. They obtained well-posedness under minimal assumptions on the regularity of the coefficient functions, again, excluding plane-wave initial conditions. Here, we study well-posedness of solutions of such a system with constant (time- and space-independent) coefficients in the whole 
space through a plane-wave synthesis and analysis. This is motivated by the calculations carried out by Richards [21] pertaining to plane-wave reflection in bimaterials with relaxation. Richards observed that in a configuration of two distinct homogeneous isotropic viscoelastic solids separated by a plane interface, at particular scattering angles plane waves will exhibit an exponentially growing behavior. We will study the stability of solutions in a generalized Zener solid with an explicit dependence on the parameters controlling the relaxation.

Solem et al. [23] considered one-dimensional linear hyperbolic systems with a stable relaxation term of rank 1 and pointed out a connection between stability properties of such systems and the theory describing general properties of polynomial roots. In particular, it was shown in [23] that strictly hyperbolic relaxation systems are linearly stable if and only if the roots of the homogeneous and equilibrium characteristic polynomials interlace on the imaginary axis. In Sec. 4 we invoke the Routh-Hurwitz theorem to determine the number of roots of the characteristic polynomial in the right half-plane and mention in Sec. 5 how the location and multiplicity of roots influence stability.

In [1] Bécache et al. defined the following quantity as the energy of the model

$$
E(q, \sigma, t)=\frac{1}{2}\|\dot{q}\|_{\rho}^{2}+\frac{1}{2}\|\epsilon(q)\|_{\mathbf{C}}^{2}+\frac{1}{2}\|s\|_{(\mathbf{D}-\mathbf{C})^{-1}}^{2}
$$

The sum of the first two terms in (1.1) corresponds to the standard energy in the purely elastic case and the final term is the norm of the difference between viscoelastic and elastic stresses. It turns out that in the absence of the source term the energy decreases in time if the absorption condition holds, i.e. $\mathbf{D}-\mathbf{C}$ is positive definite where $\mathbf{D}$ and $\mathbf{C}$ are two symmetric tensors of order four that define the constitutive law (see Ref. [1] for details and notation). In Sec. 5 we perform a similar analysis in Fourier space and comment on the conditions of energy dissipation.

\section{Memory kernels and relaxation}

For an arbitrary point $x \in \mathbb{R}^{d}$ in the medium let the vector-valued displacement of the point from its position in an undeformed state be $q(x, t)$, let $\sigma_{i j}(x, t)$ be the stress tensor with $(\nabla \cdot \sigma)_{i}=\sum_{j=1}^{d} \frac{\partial \sigma_{i j}}{\partial x_{j}}$, let $F(x, t)$ represent the external forces per unit volume and $\rho(x)$ denote the density. The description of wave propagation in a general medium is expressed by the equation of motion

$$
\rho \ddot{q}_{i}=(\nabla \cdot \sigma)_{i}+F_{i}, \quad i=1, \ldots, d
$$

which follows from the conservation of linear momentum.

The so-called Zener or standard linear solid model provides the most general linear constitutive law between the stress, strain and their rates of change

$$
\sigma+\tau_{\sigma} \dot{\sigma}=M_{R}\left(\epsilon(q)+\tau_{\epsilon} \dot{\epsilon}(q)\right)
$$

relating them by three parameters: the deformation modulus $M_{R}$, the stress relaxation time $\tau_{\sigma}$ and the strain relaxation time $\tau_{\epsilon}[11]$.

Rewriting Eq. (2.2) in the following equivalent form

$$
\partial_{t}\left(e^{t / \tau_{\sigma}} \sigma\right)=M_{R} \frac{\tau_{\epsilon}}{\tau_{\sigma}} \partial_{t}\left(e^{t / \tau_{\sigma}} \epsilon\right)+\frac{M_{R}\left(\tau_{\sigma}-\tau_{\epsilon}\right)}{\tau_{\sigma}^{2}} e^{t / \tau_{\sigma}} \epsilon
$$

and integrating it choosing the initial condition $\sigma_{0}=M_{R} \frac{\tau_{\epsilon}}{\tau_{\sigma}} \epsilon_{0}$ results in the stress-strain relation

$$
\sigma=M_{R} \frac{\tau_{\epsilon}}{\tau_{\sigma}} \epsilon+\frac{M_{R}\left(\tau_{\sigma}-\tau_{\epsilon}\right)}{\tau_{\sigma}^{2}} \int_{0}^{t} e^{-(t-s) / \tau_{\sigma}} \epsilon(s) d s
$$


The first term on the right-hand side of (2.3) represents Hooke's law and the second term indicates that the stress at any given instance depends upon the strain at all preceding times. The idea that stress depends both on the present and past value of strain is attributed to Boltzmann. Early contributions are also due to Maxwell, Kelvin and Voigt [20].

Dividing both sides of (2.1) by the density and taking the divergence results in

$$
\phi_{t t}=\nabla \cdot\left(\frac{1}{\rho} \nabla \cdot \sigma\right)+f
$$

where $f=\nabla \cdot(F / \rho)$ and $\phi=\nabla \cdot q$ are scalar-valued functions. Substituting Eq. (2.3) into to the above equation and remembering that the strain tensor and the displacement vector satisfy $\epsilon_{i j}=1 / 2\left(\partial q_{i} / \partial x_{j}+\partial q_{j} / \partial x_{i}\right)$ we arrive at the second-order integro-differential equation modeling viscoelastic motion

$$
\phi_{t t}=\nabla \cdot\left(c^{2}(x) \nabla \phi\right)+\int_{0}^{t} \nabla \cdot\left(a(x) e^{-(t-s) / \tau_{\sigma}} \nabla \phi(x, s)\right) d s+f,
$$

where $c^{2}(x)=\frac{2 \mu+\lambda}{\rho} \frac{\tau_{\epsilon}}{\tau_{\sigma}}, a(x)=\frac{2 \mu+\lambda}{\rho} \frac{\left(\tau_{\sigma}-\tau_{\epsilon}\right)}{\tau_{\sigma}^{2}}$ and deformation modulus is written in terms of the Lame parameters, that is $M_{R}=2 \mu+\lambda$.

When an elastic body is under the effect of hydrostatic pressure, i.e. when a pressure of the same magnitude acts on every unit area on the surface of the body, both the strain and stress tensors are determined by their diagonal components. In fact, if $p(x, t)$ is the pressure field, then $\sigma_{i j}=-p \delta_{i j}$. In this case a derivation similar to the one carried out above yields a scalar wave equation for $p=-1 / 3 \operatorname{tr}(\sigma)$ describing the propagation of acoustic waves in a viscoelastic fluid [18] (see also [4] for the derivation of a scalar wave equation for the trace of the strain tensor or the dilatation).

Quite often a combination of weightless springs and dashpots filled with viscous fluids is used as a good mechanical model that describes anelastic phenomena and the behavior of a variety of materials. A spring and a dashpot connected in series yield the Maxwell model, while being connected in parallel give the Kelvin-Voigt model. These models can be obtained from the Zener model in (2.2) by taking the limits $\tau_{\epsilon} \rightarrow \infty$ and $\tau_{\sigma} \rightarrow 0$, respectively.

The generalized Zener model consists of a number of Zener elements combined in parallel and takes into account multiple relaxation times. The total stress acting on the system is the sum of the stresses experienced by each element $\sigma=\sum_{i=1}^{k} \sigma_{i}$. Denoting the deformation moduli and relaxation times by

$$
M_{R i}=\frac{E_{1 i} E_{2 i}}{E_{1 i}+E_{2 i}}, \tau_{\sigma i}=\frac{\eta_{i}}{E_{1 i}+E_{2 i}}=\frac{1}{b_{i}}, \tau_{\epsilon i}=\frac{\eta_{i}}{E_{2 i}}, i=1,2, \ldots, k,
$$

where $E_{1 i}, E_{2 i}$ are the Young moduli of the springs in the $i$ th element and $\eta_{i}$ is the viscosity of the corresponding dashpot we arrive at the generalization of Eq. (2.4) with $c^{2}=\sum_{i=1}^{k} M_{R i} \tau_{\epsilon i} \tau_{\sigma i}^{-1}$

$$
\phi_{t t}=\nabla \cdot\left(c^{2}(x) \nabla \phi\right)+\sum_{i=1}^{k} \int_{0}^{t} \nabla \cdot\left(a_{i}(x) e^{-b_{i}(x)(t-s)} \nabla \phi(x, s)\right) d s+f .
$$


We will assume that

[A1] $c^{2}$ is positive bounded away from zero, $b_{i}>0$ are pairwise distinct and no sign condition is imposed on the coefficients $a_{i} \neq 0$, unless otherwise stated.

Since (2.5) is linear, by considering the difference of solutions we can study the effect of the sufficiently regular external force separately with zero initial conditions. Therefore, in what follows, we put $f=0$.

Initial value problem. Let $d \geq 1$ be the space dimension and $x=\left(x_{1}, x_{2}, \ldots, x_{d}\right) \in$ $\mathbb{R}^{d}$ be the space and $t \in \mathbb{R}$ the time variables. It is convenient to formulate the equation of motion derived in the previous section as a Cauchy initial value problem

$$
\begin{aligned}
\mathcal{L}\left(\partial_{t}, \nabla\right) U=\partial_{t} U+\sum_{j=1}^{d} A_{j}(x, t) \partial_{x_{j}} U+B(x, t) U & =0, \\
U(x, 0) & =U_{0},
\end{aligned}
$$

where $U: \mathbb{R}^{d} \times(0, T) \rightarrow \mathbb{R}^{n}$ is the unknown vector, $A_{j}, B: \mathbb{R}^{d} \times(0, T) \rightarrow \mathbb{M}^{n \times n}$ are matrix coefficients with $n=k d+d+1 \geq 3$ being the size of the system, and the initial datum $U_{0}: \mathbb{R}^{d} \rightarrow \mathbb{R}^{n}$ is given in a suitable function space. Using the substitution

$$
\begin{aligned}
& u=-\phi_{t}(x, t) \\
& v=c^{2}(x) \nabla \phi+\sum_{i=1}^{k} \int_{0}^{t} a_{i}(x) e^{-b_{i}(x)(t-s)} \nabla \phi(x, s) d s \\
& w_{i}=-a_{i}(x) \nabla \phi+b_{i}(x) \int_{0}^{t} a_{i}(x) e^{-b_{i}(x)(t-s)} \nabla \phi(x, s) d s, \quad i=1,2, \ldots, k, \\
& v=\left(v_{1}, v_{2}, \ldots, v_{d}\right), \quad w_{i}=\left(w_{i_{1}}, w_{i_{2}}, \ldots, w_{i_{d}}\right)
\end{aligned}
$$

Eq. (2.5) can be recast as a system

$$
\begin{aligned}
u_{t}+\nabla \cdot v & =0, \\
v_{t}+c^{2}(x) \nabla u+\sum_{i=1}^{k} w_{i} & =0, \\
\left(w_{i}\right)_{t}-a_{i}(x) \nabla u+b_{i}(x) w_{i} & =0, \quad i=1,2, \ldots, k,
\end{aligned}
$$

which can be written as

$$
\left(\begin{array}{c}
u \\
v \\
w_{1} \\
\vdots \\
w_{k}
\end{array}\right)_{t}+\underbrace{\left(\begin{array}{ccccc}
0 & \nabla & 0 & \cdots & 0 \\
c^{2} \nabla & 0 & 0 & \cdots & 0 \\
-a_{1} \nabla & 0 & 0 & \cdots & 0 \\
\vdots & \vdots & \vdots & \vdots & \vdots \\
-a_{k} \nabla & 0 & 0 & \cdots & 0
\end{array}\right)}_{A(\nabla)}\left(\begin{array}{c}
u \\
v \\
w_{1} \\
\vdots \\
w_{k}
\end{array}\right)+\underbrace{\left(\begin{array}{c}
0 \\
\sum_{i=1}^{k} w_{i} \\
b_{1} w_{1} \\
\vdots \\
b_{k} w_{k}
\end{array}\right)}_{B\left(u, v, w_{1}, \ldots, w_{k}\right)^{T}}=0 .
$$

Expanding $A(\nabla)$ as $A(\nabla)=\sum_{j=1}^{d} A_{j}(x, t) \partial_{x_{j}} \quad$ we arrive at (2.6) with $U=$ $\left(u, v_{1}, \ldots, v_{d}, w_{1_{1}}, w_{1_{2}}, \ldots, w_{k_{d}}\right)$ and $U_{0}=\left(u^{0}, v_{1}^{0}, \ldots, v_{d}^{0}, w_{1_{1}}^{0}, w_{1_{2}}^{0}, \ldots, w_{k_{d}}^{0}\right)$. 
One can recover $\phi(x, t)$ by first noting that

$$
\begin{aligned}
& \phi_{t}(x, t)=-u, \\
& \nabla \phi(x, t)=\frac{v-\sum_{i=1}^{k} \frac{w_{i}}{b_{i}}}{c^{2}+\sum_{i=1}^{k} \frac{a_{i}}{b_{i}}}
\end{aligned}
$$

and then using the fundamental theorem for gradients. The condition $c^{2}+\sum_{i=1}^{k} \frac{a_{i}}{b_{i}}>0$ is motivated on physical grounds (see assumption [A2] and Remark 4.1) and $b_{i}>0$ by assumption [A1], so $\nabla \phi$ in the second equality is well-defined.

\section{Well-posedness of the initial value problem}

For the rest of the paper we shall consider the constant-coefficient systems, i.e. assume that $A_{j}, B$ are independent of $(x, t)$ and denote the principal part of $\mathcal{L}$ given in (2.6) by

$$
L=\partial_{t}+\sum_{j=1}^{d} A_{j} \partial_{x_{j}} .
$$

The Fourier transform of (2.6) in the spatial directions gives

$$
\hat{U}_{t}+i \sum_{j=1}^{d} \xi_{j} A_{j} \hat{U}+B \hat{U}=0, \hat{U}(\xi, 0)=\hat{U}_{0}
$$

where $\xi=\left(\xi_{1}, \xi_{2}, \ldots, \xi_{d}\right) \in \mathbb{R}^{d}$ is a vector dual to $x$. Using the notation $A(\xi)=$ $\sum_{j=1}^{d} \xi_{j} A_{j}$ we can write the solution of this ordinary differential equation as $\hat{U}(\xi, t)=$ $e^{-t(B+i A(\xi))} \hat{U}_{0}(\xi)$. When $U_{0} \in H^{s}\left(\mathbb{R}^{d}\right)^{n}$, by taking the inverse Fourier transform one can show that the Cauchy problem (2.6) admits a continuous solution

$$
U(x, t)=\frac{1}{(2 \pi)^{d / 2}} \int_{\mathbb{R}^{d}} e^{i x \cdot \xi} \hat{U}(\xi, t) d \xi
$$

with values in $H^{s}$ if

$$
\sup _{\xi \in \mathbb{R}^{d}, 0 \leq t \leq T}\left\|e^{-t(B+i A(\xi))}\right\|<\infty
$$

which is equivalent (see, for example, proposition 2.I.1 in [19]) to writing

$$
\sup _{\xi \in \mathbb{R}^{d}}\left\|e^{-i A(\xi)}\right\|<\infty .
$$

Throughout this paper, we will use the matrix norm $\|M\|=\sup _{|x|=1}|M x|$ induced by the Euclidean norm. Notice that the property (3.3) does not depend on time once $t \neq 0$ since $t A(\xi)=A(t \xi)$. We can also absorb the minus sign by virtue of the change $\xi \rightarrow-\xi$.

DeFinition 3.1. The operator $L$ is called hyperbolic if the corresponding symbol $A(\xi)$ satisfies (3.3).

Proposition 3.2. Assume that [A1] holds. The matrix $A(\xi)$ is uniformly diagonalizable with real eigenvalues: There exists $P(\xi)$ such that $P(\xi) A(\xi) P^{-1}(\xi)$ is diagonal and real for all $\xi \in \mathbb{R}^{d}$ and

$$
\sup _{\xi \in \mathbb{S}^{d-1}}\left\|P^{-1}(\xi)\right\|\|P(\xi)\|<\infty
$$


Proof. A simple computation shows that the characteristic equation of $A$ splits as

$$
\lambda^{k d+d-1}\left(\lambda^{2}-c^{2}|\xi|^{2}\right)=p_{1}^{k d+d-1}(\lambda, \xi) p_{ \pm}(\lambda, \xi) .
$$

where $p_{1}(\lambda, \xi)=\lambda$ and $p_{ \pm}(\lambda, \xi)=\lambda^{2}-c^{2}|\xi|^{2}$. Observe that $p_{ \pm}$and $p_{1}$ are homogeneous polynomials in $\lambda,|\xi|$ and $\lambda$, respectively, with real and simple roots, and they have no common root for $\xi \in \mathbb{R}^{d} \backslash\{0\}$. Let

$$
E_{j}=\prod_{i \neq j} \frac{A-\lambda_{i} I_{n}}{\lambda_{j}-\lambda_{i}}, \quad \text { for } j=1,2,3 \text { and } n=k d+d+1
$$

with $\lambda_{1}=0$ and $\lambda_{2,3}= \pm c|\xi|$. One can check that $E_{j}$ 's are mutually orthogonal and complete in the sense that $E_{i} E_{j}=\delta_{i j} E_{j}$ and $\sum_{j=1}^{3} E_{j}=I_{n}$ and verify that the following decomposition takes place

$$
A(\xi)=\sum_{j=1}^{3} \lambda_{j} E_{j}
$$

Next we define a positive-definite matrix $H(\xi)=\sum_{j} E_{j}^{T} E_{j}$ which admits a unique square root. Then, since $A(\xi)^{T}=\sum_{j=1}^{3} \lambda_{j} E_{j}^{T}$, it follows that $H(\xi) A(\xi)=A(\xi)^{T} H(\xi)$ which implies that $H^{1 / 2}(\xi) A(\xi) H^{-1 / 2}(\xi)$ is symmetric and diagonalizable in an orthonormal basis. Hence $A(\xi)=P^{-1}(\xi) D(\xi) P(\xi)$ where $D(\xi)$ is diagonal with real eigenvalues and $P(\xi)=O(\xi) H^{1 / 2}(\xi)$ with an orthogonal matrix $O(\xi)$.

It remains to show that $P(\xi)$ is uniformly bounded. We note that

$$
|y|^{2}=\left|\sum_{j=1}^{3} E_{j} y\right|^{2} \leq 3 \sum_{j=1}^{3}\left|E_{j} y\right|^{2}=3\left|H^{1 / 2} y\right|^{2}
$$

and thus $\left\|H^{-1 / 2}(\xi)\right\| \leq \sqrt{3}$.

Using the Lagrange multiplier method with the constraint $|x|=1$ or calculating the largest eigenvalue of $E_{j}^{T} E_{j}$ we find that

$$
\begin{aligned}
& \left\|E_{1}\right\|^{2}=1+\frac{1}{c^{4}} \sum_{i=1}^{k} a_{i}^{2}, \\
& \left\|E_{2}\right\|^{2}=\left\|E_{3}\right\|^{2}=\frac{1+c^{2}}{4}\left(\frac{1}{c^{2}}+1+\frac{1}{c^{4}} \sum_{i=1}^{k} a_{i}^{2}\right),
\end{aligned}
$$

are independent of $\xi$, and therefore

$$
\left|H^{1 / 2} y\right|^{2}=\sum_{j=1}^{3}\left|E_{j} y\right|^{2} \leq\left(\left\|E_{1}\right\|^{2}+\left\|E_{2}\right\|^{2}+\left\|E_{3}\right\|^{2}\right)|y|^{2} \leq \frac{C^{2}}{3}|y|^{2} .
$$

We conclude that the diagonalization is well-conditioned because

$$
\left\|P^{-1}(\xi)\right\|\|P(\xi)\|=\left\|H^{-1 / 2}(\xi)\right\|\left\|H^{1 / 2}(\xi)\right\| \leq C
$$


independently of $\xi$.

Definition 3.3. The Cauchy problem for a constant coefficient operator $\mathcal{L}$ is weakly (strongly) well-posed if for any initial data $U_{0} \in H^{s}\left(\mathbb{R}^{d}\right)$ with $s>0 \quad(s=0)$, there is a unique solution $U(t) \in \mathcal{C}\left(\mathbb{R}^{+}, H^{s}\left(\mathbb{R}^{d}\right)\right)$ that satisfies

$$
\|U(t)\|_{L^{2}\left(\mathbb{R}^{d}\right)} \leq K e^{\alpha t}\left\|U_{0}\right\|_{H^{s}\left(\mathbb{R}^{d}\right)}, \quad t \geq 0,
$$

with $K>0$ and $\alpha \in \mathbb{R}$ independent of time.

LEMma 3.4. (Strang, [25]) If $\left\|e^{t A}\right\| \leq C$ for $t \geq 0$, then $\left\|e^{t(A+B)}\right\| \leq C e^{t C\|B\|}$.

Proof. This is an exponential analogue of another lemma due to Strang [24] which states that if $\left\|M^{n}\right\| \leq C$ for $n \geq 0$, then $\left\|(M+R)^{n}\right\| \leq C e^{n C\|R\|}$. Setting $M=e^{\varepsilon A}$ and $R=e^{\varepsilon(A+B)}-M$ with sufficiently small $\varepsilon$ we have $\left\|M^{n}\right\| \leq C$ for $n \geq 0$ and hence

$$
\left\|(M+R)^{n}\right\|=\left\|e^{n \varepsilon(A+B)}\right\| \leq C e^{n C\|R\|}
$$

Let $n$ tend to infinity, while keeping $t=n \varepsilon$ fixed. In this limit we have $n R \rightarrow t B$ and the lemma follows.

TheOREm 3.5. Assume that [A1] holds. The operator $L$ is hyperbolic and the Cauchy problem for a constant coefficient operator $\mathcal{L}$ is strongly well-posed.

Proof. By Proposition 3.2, we have that for all $\xi \in \mathbb{R}^{d}$ and $t \geq 0$

$$
\left\|e^{i t A(\xi)}\right\| \leq\left\|P^{-1} e^{i t D} P\right\| \leq C
$$

where $D$ is diagonal with real entries, $e^{i t D}$ is unitary and therefore leaves the matrix norm invariant. Hence $L$ is hyperbolic.

Using (3.2), Parseval's relation and hyperbolicity of $L$ we obtain the following estimate

$$
\begin{aligned}
\|U(t)\|_{L^{2}\left(\mathbb{R}^{d}\right)} & =\left\|e^{-t(B+i A(\xi))} \hat{U}_{0}(\xi)\right\|_{L^{2}\left(\mathbb{R}^{d}\right)} \\
& \leq\left\|e^{-t(B+i A(\xi))}\right\|\left\|\hat{U}_{0}(\xi)\right\|_{L^{2}\left(\mathbb{R}^{d}\right)} \leq C e^{t C\|B\|}\left\|U_{0}\right\|_{L^{2}\left(\mathbb{R}^{d}\right)}
\end{aligned}
$$

completing the claim. The last inequality follows from Lemma 3.4. Note that since $A$ and $B$ do not commute, it does not hold that $\left\|e^{t(A+B)}\right\|=\left\|e^{t A} e^{t B}\right\|$ for $t>0$.

REMARK 3.1. 1. If $L$ is hyperbolic and $U_{0} \in H^{s}\left(\mathbb{R}^{d}\right)^{n}$, then application of Gronwall's inequality shows that there is a continuous solution with values in $H^{s}$ if one has a variable-coefficient lower order term $B(x) \in L^{\infty}\left(\mathbb{R}^{d}\right)$. In this case the Cauchy problem for $L+B$ is also strongly well-posed. Hyperbolicity and well-posedness is a property of $A$ alone.

2. In the notation of Def. 1.1 we have $\lambda_{1}=-c|\xi|, \lambda_{2}=0$ and $\lambda_{3}=c|\xi|$ with $l_{1}=1$, $l_{2}=k d+d-1$ and $l_{3}=1$. Operator $L$ is constantly hyperbolic, that is, the symbol $A(\xi)$ is diagonalizable with real eigenvalues and the algebraic multiplicities of eigenvalues remain constant as $\xi$ ranges along $\mathbb{S}^{d-1}$. Strict or constant hyperbolicity implies hyperbolicity [2].

In [14] Métivier provided a few examples of systems satisfying the block structure condition, including the equations of linear elasticity. Our results show that this important class of systems can be enlarged by the generalized Zener model of viscoelasticity. 
3. The matrices $A_{j}$ do not commute, i.e. $A_{j} A_{i} \neq A_{i} A_{j}$ for $i \neq j$. Hence they cannot be simultaneously diagonalized and Eq. (3.1) cannot be transformed to a system consisting of $n$ uncoupled scalar equations.

4. Eq. (3.1) can be viewed as a linearization of a system with a non-linear source term $Q(U)$ about a constant state in equilibrium. Typically, the source term is divided by a small parameter that determines the rate of relaxation towards equilibrium. To ensure the existence of a well-behaved zero relaxation limit, Yong [26] introduced the so-called stability criterion which necessitates that there is $C(U)>0$ such that

$$
\left\|e^{\delta Q_{U}(U)+i A(\xi)}\right\| \leq C(U)
$$

for all $\delta \geq 0, \xi \in \mathbb{R}^{d}$ with $\{U: Q(U)=0\} \neq \emptyset$. Here, $Q_{U}(U)$ denotes the Jacobian matrix of the source term. This criterion is somewhat stronger than the hyperbolicity condition and reduces to that when $\delta=0$ ( $c f$. Eq. (3.3) and the inequality preceding it).

\section{Plane-wave analysis}

Waves at a sufficiently large distance from the source behave locally like plane waves. This motivates one to study the behavior of plane waves as possible growth modes in the system under consideration.

THEOREM 4.1. Let $A_{j}, B$ be constant-coefficient matrices and $d \geq 1$. The eigenvalues of $\Phi(i \xi)=-(B+i A(\xi))$ are roots of the characteristic polynomial

$$
\tilde{p}\left(\lambda, \xi_{1}, \ldots, \xi_{d}\right)=p(\lambda,|\xi|) \lambda^{d-1} \prod_{i=1}^{k}\left(\lambda+b_{i}\right)^{d-1}
$$

where $p(\lambda,|\xi|)$ is the characteristic polynomial corresponding to the system derived from the one-dimensional wave equation with $k$ memory terms and $\xi \in \mathbb{R}$ replaced by $|\xi|=$ $\sqrt{\xi_{1}^{2}+\cdots+\xi_{d}^{2}} \in \mathbb{R}:$

$$
p(\lambda, \xi)=(-1)^{k}\left(\lambda^{2}+c^{2} \xi^{2}+\xi^{2} \sum_{i=1}^{k} \frac{a_{i}}{\lambda+b_{i}}\right) \prod_{i=1}^{k}\left(\lambda+b_{i}\right)
$$

Proof. Perform the following similarity transformation: pre-multiply $\lambda I_{k d+d+1}-$ $\Phi(i \xi)$ by the block diagonal matrix

$$
S=\left(\begin{array}{ccccc}
|\xi| & 0 & \ldots & \ldots & 0 \\
0 & \Xi_{1} & 0 & \ldots & 0 \\
0 & 0 & \ddots & \ddots & \vdots \\
\vdots & \vdots & \ddots & \ddots & 0 \\
0 & 0 & \ldots & 0 & \Xi_{k+1}
\end{array}\right), \text { where } \Xi_{j}=\left(\begin{array}{ccccc}
\xi_{1} & \xi_{2} & \xi_{3} & \ldots & \xi_{d} \\
0 & \xi_{2} & \xi_{3} & \ldots & \xi_{d} \\
0 & 0 & \ddots & \ddots & \vdots \\
\vdots & \vdots & \ddots & \ddots & \xi_{d} \\
0 & 0 & \ldots & 0 & \xi_{d}
\end{array}\right)
$$

are identical for all $1 \leq j \leq k+1$, and post-multiply by its inverse $S^{-1}$. Successively develop the resulting determinant by the columns containing a single non-zero element thereby accounting for the factor $\lambda^{d-1} \prod_{i=1}^{k}\left(\lambda+b_{i}\right)^{d-1}$ in Eq. (4.1).

Without loss of generality, we assume that $0<b_{1}<b_{2}<\cdots<b_{k}$ and consider $g(\lambda)=(-1)^{k} p(\lambda, \xi)$. Since the characteristic polynomial in higher dimensions splits as 
in (4.1), it suffices to analyze the roots of $p(\lambda, \xi)$.

Proposition 4.2 (All $a$ 's are negative). Let $d=1, \xi \neq 0$ and $a_{i}<0$ for all $1 \leq i \leq k$, then i) if $g(0)>0$, all eigenvalues of $\Phi(i \xi)$ have negative real parts; ii) if $g(0)=0$, then one eigenvalue is zero and the rest have negative real parts; iii) if $g(0)<0$, then only one eigenvalue of $\Phi(i \xi)$ is positive and all other eigenvalues have negative real parts.

Proof. If $\xi=0$, the eigenvalues are $-b_{i}<0$ and 0 with multiplicity 2 .

If $\xi \neq 0$, the function $g(\lambda) / \prod_{i=1}^{k}\left(\lambda+b_{i}\right)$ has $k$ simple poles at $-b_{i}$ and since all $a_{i}$ 's have the same sign, there are $k-1$ real roots $r_{i}$ of $p(\lambda, \xi)$ between these poles. from

i) Assume that $g(0)>0$, then a further real root lies between 0 and $-b_{1}$, as follows

$$
g\left(-b_{1}\right) g(0)=\xi^{2} a_{1} \prod_{i \neq 1}\left(b_{i}-b_{1}\right) g(0)<0
$$

and the Intermediate value theorem. The function $g(\lambda)$ can now be factored as

$$
\left(\lambda^{2}+\alpha \lambda+\beta\right) \prod_{i=1}^{k}\left(\lambda-r_{i}\right)=0 .
$$

The coefficients $\alpha, \beta$ are real since $r_{i}$ 's are real for all $1 \leq i \leq k$. Denote by $r_{k+1}$ and $r_{k+2}$ the two roots (real or complex conjugate) of $\lambda^{2}+\alpha \lambda+\beta$, then by Vieta's theorem,

$$
\begin{aligned}
& r_{k+2}+r_{k+1}=-\sum_{i=1}^{k} b_{i}-\sum_{i=1}^{k} r_{i}<0, \\
& r_{k+2} \cdot r_{k+1}=g(0) / \prod_{i=1}^{k}\left|r_{i}\right|>0 .
\end{aligned}
$$

If $r_{k+1}$ and $r_{k+2}$ are complex conjugate, then $\Re\left(r_{k+1}\right)=\Re\left(r_{k+2}\right)<0$. If $r_{k+1}$ and $r_{k+2}$ are real, then $r_{k+1}<0$ and $r_{k+2}<0$. The same result was obtained in [13].

ii) If $g(0)=0$, then in addition to a real root $r_{i}$ between each consecutive $-b_{i}$ 's, there is a zero eigenvalue since the constant term in $p(\lambda, \xi)$ is absent and therefore one can factor out $\lambda$ :

$$
\lambda\left(\lambda^{2}+\alpha \lambda+\beta\right) \prod_{i=1}^{k-1}\left(\lambda-r_{i}\right)=0
$$

By Vieta's theorem

$$
\begin{aligned}
& r_{k+2}+r_{k+1}=-\sum_{i=1}^{k} b_{i}-\sum_{i=1}^{k-1} r_{i}<0, \\
& r_{k+2} \cdot r_{k+1}=g^{\prime}(0) / \prod_{i=1}^{k-1}\left|r_{i}\right|=-\xi^{2}\left(\sum_{i=1}^{k} \frac{a_{i}}{b_{i}^{2}}\right) \prod_{i=1}^{k} b_{i} / \prod_{i=1}^{k-1}\left|r_{i}\right|>0
\end{aligned}
$$

where $r_{k+1}$ and $r_{k+2}$ are roots (real or complex conjugate) of $\lambda^{2}+\alpha \lambda+\beta$ and we used $g(0)=0$ in the expression for $g^{\prime}(0)$. As in case i) above, $r_{k+1}$ and $r_{k+2}$ have negative real parts. 
iii) Now assume that $g(0)<0$. Since there are $k-1$ real roots $r_{i}$ between $k$ simple poles $-b_{i}, g(\lambda)$ can be written as

$$
\left(\lambda^{3}+\alpha \lambda^{2}+\beta \lambda+\gamma\right) \prod_{i=1}^{k-1}\left(\lambda-r_{i}\right)=0
$$

By Vieta's theorem

$$
\begin{aligned}
& r_{k+1}+r_{k+2}+r_{k}=-\sum_{i=1}^{k} b_{i}-\sum_{i=1}^{k-1} r_{i}<0, \\
& r_{k+1} \cdot r_{k+2} \cdot r_{k}=(-1)^{k+2} g(0) / \prod_{i=1}^{k-1} r_{i}=-g(0) / \prod_{i=1}^{k-1}\left|r_{i}\right|>0
\end{aligned}
$$

where $r_{k}, r_{k+1}, r_{k+2}$ are roots of the cubic equation. An algebraic equation of an odd degree and real coefficients must posses at least one real root. Eq. (4.2) implies that this root is positive. The other two roots of the cubic equation have negative real parts.

Proposition 4.3 (All $a$ 's are positive). Let $d=1, \xi \neq 0$ and $a_{i}>0$ for all $1 \leq i \leq$ $k$, then two eigenvalues of $\Phi(i \xi)$ have positive real parts and the others are real and negative.

Proof. If $\xi=0$, the eigenvalues are $-b_{i}<0$ and zero (two-fold).

If $\xi \neq 0$, then the $k-1$ real roots $r_{i}$ of $p(\lambda, \xi)$ strictly interlace $-b_{i}$ for $1 \leq i \leq k$. By the Intermediate value theorem there is also a root to the left of $-b_{k}=-\max _{i} b_{i}$ since $\lim _{\lambda \rightarrow-\infty} p(\lambda, \xi)=+\infty$ and $p\left(-b_{k}, \xi\right)=-\xi^{2} a_{k} \prod_{i \neq k}\left(b_{k}-b_{i}\right)<0$. Thus for some real $\alpha, \beta$ we have the factorization

$$
\left(\lambda^{2}+\alpha \lambda+\beta\right) \prod_{i=1}^{k}\left(\lambda-r_{i}\right)=0 .
$$

By Vieta's theorem the roots $r_{k+1}, r_{k+2}$ of $\lambda^{2}+\alpha \lambda+\beta$ satisfy

$$
\begin{aligned}
& r_{k+2}+r_{k+1}=-\sum_{i=1}^{k} b_{i}-\sum_{i=1}^{k} r_{i}>0 \\
& r_{k+2} \cdot r_{k+1}=g(0) / \prod_{i=1}^{k}\left|r_{i}\right|>0
\end{aligned}
$$

where $g(0)>0$, since $a_{i}>0$ for all $1 \leq i \leq k$. In fact, $g(\lambda)>0$ holds for $\lambda \geq 0$, so $r_{k+1}$ and $r_{k+2}$ cannot be real and positive. Hence they are complex conjugate with positive real parts.

For each $1 \leq i \leq k-1$, the signs of $a_{i}$ and $a_{i+1}$ determine the number of jumps of the rational function $g(\lambda) / \prod_{i=1}^{k}\left(\lambda+b_{i}\right)$ from $\pm \infty$ to $\mp \infty$ as the argument changes from $-b_{i+1}$ to $-b_{i}$. If the signs of $a_{i}$ and $a_{i+1}$ are the same as in Propositions 4.2 and 4.3 , then there is a real root between $-b_{i+1}$ and $-b_{i}$ corresponding to a jump from $\pm \infty$ to $\mp \infty$. This greatly simplifies the problem of root location which becomes increasingly complicated if the signs of the $a_{i}$ 's are arbitrary, as can already be seen from the simplest example with $k=2$. In this case, the characteristic polynomial takes 
the form

$$
\begin{aligned}
g(\lambda)=p(\lambda, \xi) & =\lambda^{4}+\left(b_{1}+b_{2}\right) \lambda^{3}+\left(c^{2} \xi^{2}+b_{1} b_{2}\right) \lambda^{2} \\
& +\xi^{2}\left(a_{1}+c^{2} b_{1}+a_{2}+c^{2} b_{2}\right) \lambda+\xi^{2}\left(c^{2} b_{1} b_{2}+a_{1} b_{2}+a_{2} b_{1}\right)
\end{aligned}
$$

Let $\Delta_{i}$ denote the $i$ th Hurwitz determinant obtained from the coefficients of the characteristic equation, so that

$$
\begin{aligned}
& \Delta_{1}=b_{1}+b_{2}, \Delta_{2}=\left|\begin{array}{cc}
b_{1}+b_{2} & g^{\prime}(0) \\
1 & c^{2} \xi^{2}+b_{1} b_{2}
\end{array}\right|, \Delta_{3}=\left|\begin{array}{ccc}
b_{1}+b_{2} & g^{\prime}(0) & 0 \\
1 & c^{2} \xi^{2}+b_{1} b_{2} & g(0) \\
0 & b_{1}+b_{2} & g^{\prime}(0)
\end{array}\right|, \\
& \Delta_{4}=\left|\begin{array}{cccc}
b_{1}+b_{2} & g^{\prime}(0) & 0 & 0 \\
1 & c^{2} \xi^{2}+b_{1} b_{2} & g(0) & 0 \\
0 & b_{1}+b_{2} & g^{\prime}(0) & 0 \\
0 & 1 & c^{2} \xi^{2}+b_{1} b_{2} & g(0)
\end{array}\right|=g(0) \Delta_{3} .
\end{aligned}
$$

Proposition 4.4. Let $d=1, \xi \neq 0$ and $a_{1} a_{2}<0$, then

- i) when $g(0)>0$ all eigenvalues have negative real parts if $\Delta_{2}>0$ and $\Delta_{3}>0$, otherwise two roots have negative real parts and two roots have non-negative real parts;

- ii) a) when $g(0)=0$ and $g^{\prime}(0)>0$, one root is zero and three have negative real parts if $\Delta_{2}>0$, otherwise there is one zero and one negative root and two roots with non-negative real parts; b) when $g(0)=0$ and $g^{\prime}(0)=0$, two roots with negative real parts and zero (two-fold); c) when $g(0)=0$ and $g^{\prime}(0)<0$, there is a zero and a positive root and two roots with negative real parts;

- iii) when $g(0)<0$, one root is negative and three roots have positive real parts if $\Delta_{2}<0$ and $\Delta_{3}<0$, otherwise one root is positive and three roots have nonpositive real parts.

Proof. If $\xi=0$, the eigenvalues are $-b_{1},-b_{2}$ and zero (two-fold).

i) Let $\xi \neq 0$ and assume $g(0)>0$. If $\Delta_{4} \neq 0$, according to the Routh-Hurwitz theorem [6] the number of roots of $g(\lambda)$ in the right half-plane $\Re(\lambda)>0$ is determined by the number of variations of sign in the sequence

$$
\left\{1, \Delta_{1}, \frac{\Delta_{2}}{\Delta_{1}}, \frac{\Delta_{3}}{\Delta_{2}}, \frac{\Delta_{4}}{\Delta_{3}}\right\}=\left\{1, b_{1}+b_{2}, \frac{\Delta_{2}}{b_{1}+b_{2}}, \frac{\Delta_{3}}{\Delta_{2}}, g(0)\right\}
$$

Hence all the roots of $g(\lambda)$ have negative real parts if and only if $\Delta_{2}>0$ and $\Delta_{3}>0$. As long as $\Delta_{4} \neq 0$, in all other cases including the singular case $\Delta_{2}=0$ there are exactly two variations of sign and therefore two roots with positive real parts, say $r_{1}$ and $r_{2}$. For some real $\alpha, \beta$ we can write

$$
\left(\lambda^{2}+\alpha \lambda+\beta\right) \prod_{i=1}^{2}\left(\lambda-r_{i}\right)=0
$$

By Vieta's theorem the roots $r_{3}, r_{4}$ of $\lambda^{2}+\alpha \lambda+\beta$ satisfy

$$
\begin{aligned}
& r_{3}+r_{4}=-\left(b_{1}+b_{2}+r_{1}+r_{2}\right)<0, \\
& r_{3} \cdot r_{4}=g(0) / r_{1} r_{2}>0
\end{aligned}
$$


i.e. $r_{3}$ and $r_{4}$ have negative real parts. Moreover, if $a_{1}<0$ and $a_{2}>0$, these roots are real: one root lies between $-b_{1}$ and 0 since $g\left(-b_{1}\right) g(0)=\xi^{2} a_{1}\left(b_{2}-b_{1}\right) g(0)<0$ and the other is to the left of $-b_{2}$ because $g\left(-b_{2}\right)=-\xi^{2} a_{2}\left(b_{2}-b_{1}\right)<0$ and $\lim _{\lambda \rightarrow-\infty} g(\lambda)=+\infty$.

If $\Delta_{4}=0$, then $\Delta_{3}=0=g^{\prime}(0) \Delta_{2}-g(0)\left(b_{1}+b_{2}\right)^{2}$. Evaluating $\Delta_{2}$ from the latter equation and comparing it with the original definition of $\Delta_{2}$, we can conclude that $\Delta_{2}>0$ and $g^{\prime}(0)>0$. In this case the polynomial enjoys the following factorization

$$
\left(\lambda^{2}+\frac{g^{\prime}(0)}{b_{1}+b_{2}}\right)\left(\lambda^{2}+\left(b_{1}+b_{2}\right) \lambda+\frac{\Delta_{2}}{b_{1}+b_{2}}\right)=0
$$

Hence there is a pair of conjugate pure imaginary roots $\pm i \sqrt{g^{\prime}(0) /\left(b_{1}+b_{2}\right)}$ and two roots with negative real parts.

ii) Assume $g(0)=0$ and $\xi \neq 0$. Since the constant term is absent, we can factor out $\lambda$ and reduce the problem of root location for $g(\lambda)$ to that for which the last Hurwitz determinant is $\Delta_{3}=g^{\prime}(0) \Delta_{2}$. a) When $g^{\prime}(0)>0$ the sequence $\left\{1, b_{1}+b_{2}, \frac{\Delta_{2}}{b_{1}+b_{2}}, g^{\prime}(0)\right\}$ has no sign variation if $\Delta_{2}>0$ and hence no roots of $\lambda^{3}+\left(b_{1}+b_{2}\right) \lambda^{2}+\left(c^{2} \xi^{2}+b_{1} b_{2}\right) \lambda+$ $g^{\prime}(0)=0$ are in the right-half plane. If $\Delta_{2}<0$, there are two sign variations and hence two roots with positive real parts and one negative root. If $\Delta_{2}=0$, the roots are $-\left(b_{1}+b_{2}\right)$ and $\pm i \sqrt{g^{\prime}(0) /\left(b_{1}+b_{2}\right)}$; b) When $g^{\prime}(0)=0$, one can factor out $\lambda$ again and obtain a quadratic equation whose roots have negative real parts; c) When $g^{\prime}(0)<0$, then $\Delta_{2}>0$. There is one positive root, say $r_{1}$, corresponding to a single sign variation in the sequence $\left\{1, b_{1}+b_{2}, \frac{\Delta_{2}}{b_{1}+b_{2}}, g^{\prime}(0)\right\}$. By Vieta's theorem the remaining two roots satisfy

$$
\begin{aligned}
& r_{2}+r_{3}=-\left(b_{1}+b_{2}+r_{1}\right)<0, \\
& r_{2} \cdot r_{3}=-g^{\prime}(0) / r_{1}>0
\end{aligned}
$$

i.e. $r_{2}$ and $r_{3}$ have negative real parts.

iii) Assume that $g(0)<0$ and $\xi \neq 0$. If $\Delta_{4} \neq 0, \Delta_{2}<0$ and $\Delta_{3}<0$ by the RouthHurwitz theorem there are three roots in the right-half plane. Since in this case we also have $g^{\prime}(0)=\frac{\Delta_{3}+g(0)\left(b_{1}+b_{2}\right)^{2}}{\Delta_{2}}>0$, it follows from the Descartes' rule of signs that only one of those three roots is real. In other cases where $\Delta_{4} \neq 0$, including the singular case $\Delta_{2}=0$, there is only one variation of sign in the sequence $\left\{1, \Delta_{1}, \frac{\Delta_{2}}{\Delta_{1}}, \frac{\Delta_{3}}{\Delta_{2}}, \frac{\Delta_{4}}{\Delta_{3}}\right\}$ and hence only one root in the right-half plane.

If $\Delta_{4}=0$, then $\Delta_{3}=0$ and $g^{\prime}(0) \Delta_{2}<0$. From the factorization

$$
\left(\lambda^{2}+\frac{g^{\prime}(0)}{b_{1}+b_{2}}\right)\left(\lambda^{2}+\left(b_{1}+b_{2}\right) \lambda+\frac{\Delta_{2}}{b_{1}+b_{2}}\right)=0
$$

we conclude that there is a pair of conjugate pure imaginary roots $\pm i \sqrt{g^{\prime}(0) /\left(b_{1}+b_{2}\right)}$ and a pair of real roots of opposite sign if $g^{\prime}(0)>0$ and $\Delta_{2}<0$. If $g^{\prime}(0)<0$ and $\Delta_{2}>0$ we have $\pm \sqrt{\left|g^{\prime}(0)\right| /\left(b_{1}+b_{2}\right)}$ and two roots with negative real parts.

Proposition 4.4 exhausts all the possibilities for the fourth order monic polynomial. When more than two $a_{i}$ 's have arbitrary signs, eigenvalues can be studied in a similar manner using higher order Hurwitz determinants even if some of those determinants vanish.

REMARK 4.1. 1. Requiring all the $a_{i}$ 's to be negative is equivalent to saying that the relaxation kernel $K(t)=-\sum_{i=1}^{k} a_{i} e^{-b_{i} t}$ is a totally monotone function. 
2. Recall that when deriving the model equation we identified $a_{i}, b_{i}$ and $c^{2}$ with the physical parameters of the system, namely, $b_{i}=\tau_{\sigma i}^{-1}>0, c^{2}=\sum_{i=1}^{k} M_{R i} \tau_{\epsilon i} \tau_{\sigma i}^{-1}>0$ and $a_{i}=M_{R i}\left(1-\tau_{\epsilon i} \tau_{\sigma i}^{-1}\right) b_{i}<0$. Since

$$
g(0)=\xi^{2}\left(c^{2}+\sum_{i=1}^{k} \frac{a_{i}}{b_{i}}\right) \prod_{i=1}^{k} b_{i}=\xi^{2} \prod_{i=1}^{k} b_{i} \sum_{i=1}^{k} M_{R i}>0,
$$

case iii) of Proposition 4.2 yielding a positive eigenvalue is unphysical. In contrast, $g(0)>0$ is fulfilled in Proposition 4.3, but it is assumed that $a_{i}>0$ (no dissipation), so a pair of complex conjugate roots with positive real parts is also unphysical.

3. The Routh-Hurwitz theorem provides necessary and sufficient conditions for all of the roots of a polynomial with real coefficients to lie in the left-half of the complex plane. It allows one to locate the roots just by employing the coefficients of the polynomial which are functions of the parameters controlling the relaxation.

4. Algebraic multiplicities of eigenvalues $\lambda_{j}(\xi)$ remain constant as $\xi$ ranges along $\mathbb{S}^{d-1}$ and $\lambda_{j}(\xi)$ are analytic functions away from the origin, admitting a power series expansion in $\xi$. This fact will be used in Proposition 4.5 below to investigate the limiting behavior of the eigenvalues as $|\xi| \rightarrow 0$ and $|\xi| \rightarrow \infty$.

Proposition 4.5. Let $d=1$ and $\lambda_{j}(i \xi)$ for $1 \leq j \leq k+2$ be the eigenvalues of $\Phi(i \xi)$, then as $|\xi| \rightarrow 0$,

$$
\Re\left(\lambda_{j}(i \xi)\right)= \begin{cases}-b_{j}-\xi^{2} \frac{a_{j}}{b_{j}^{2}}+O\left(\xi^{4}\right) & \text { for } j=1,2, \ldots, k \\ \pm \Re\left(i \xi \sqrt{c^{2}+\sum_{i=1}^{k} \frac{a_{i}}{b_{i}}}\right)+\xi^{2} \sum_{i=1}^{k} \frac{a_{i}}{2 b_{i}^{2}}+O\left(\xi^{3}\right) & \text { for } j=k+1, k+2\end{cases}
$$

if $c^{2}+\sum_{i=1}^{k} \frac{a_{i}}{b_{i}} \neq 0$ or

$$
\Re\left(\lambda_{j}(i \xi)\right)= \begin{cases}-b_{j}-\xi^{2} \frac{a_{j}}{b_{j}^{2}}+O\left(\xi^{4}\right) & \text { for } j=1,2, \ldots, k \\ \xi^{2} \sum_{i=1}^{k} \frac{a_{i}}{b_{i}^{2}}+O\left(\xi^{4}\right) & \text { for } j=k+1 \\ 0+O\left(\xi^{4}\right) & \text { for } j=k+2\end{cases}
$$

otherwise, and as $|\xi| \rightarrow \infty$

$$
\Re\left(\lambda_{j}(i \xi)\right)= \begin{cases}\Re\left(r_{j}\right)+O\left(\xi^{-1}\right) & \text { for } j=1,2, \ldots, k \\ \frac{1}{2 c^{2}} \sum_{i=1}^{k} a_{i}+O\left(\xi^{-2}\right) & \text { for } j=k+1, k+2\end{cases}
$$

Proof. Let $\zeta=i \xi \in \mathbb{C}$ and recall that $\Phi(\zeta)=-(B+\zeta A)$. Following Kato [7], [8] we treat $-B$ as an unperturbed matrix subjected to a small perturbation $-\zeta A$. The characteristic equation of $\Phi(\zeta)$ is an algebraic equation in $\lambda$ of degree $k+2$ and its roots are branches of analytic functions of $\zeta$ with only algebraic singularities. Hence, in the neighborhood of $\zeta=0$ the following expansion is valid:

$$
\lambda_{j}(\zeta)=\lambda_{j}^{(0)}+\zeta \lambda_{j}^{(1)}+\zeta^{2} \lambda_{j}^{(2)}+\ldots
$$

for $1 \leq j \leq k+2$. Here $\lambda_{j}^{(0)}$ are the eigenvalues of the unperturbed matrix $-B$ and satisfy the equation

$$
\left.p(\lambda,-i \zeta)\right|_{\zeta=0}=(-1)^{k}\left(\lambda^{(0)}\right)^{2} \prod_{i=1}^{k}\left(\lambda^{(0)}+b_{i}\right)=0
$$


so that

$$
\lambda_{j}^{(0)}= \begin{cases}-b_{j} & \text { for } j=1,2, \ldots, k \\ 0 & \text { for } j=k+1, k+2\end{cases}
$$

Solving

$$
\left.(-1)^{k} \frac{d p}{d \zeta}\right|_{\zeta=0}=\lambda^{(0)} \lambda^{(1)} \prod_{i=1}^{k}\left(\lambda^{(0)}+b_{i}\right)\left(2+\sum_{i=1}^{k} \frac{\lambda^{(0)}}{\lambda^{(0)}+b_{i}}\right)=0 \quad \text { and }\left.\quad \frac{d^{2} p}{d \zeta^{2}}\right|_{\zeta=0}=0
$$

one obtains

$$
\lambda_{j}^{(1)}= \begin{cases}0 & \text { for } j=1,2, \ldots, k \\ \pm \sqrt{c^{2}+\sum_{i=1}^{k} \frac{a_{i}}{b_{i}}} & \text { for } j=k+1, k+2\end{cases}
$$

The next order correction comes from solving $\left.\frac{d^{2} p}{d \zeta^{2}}\right|_{\zeta=0}=0$ and $\left.\frac{d^{3} p}{d \zeta^{3}}\right|_{\zeta=0}=0$, thus

$$
\lambda_{j}^{(2)}=\left\{\begin{array}{ll}
\frac{a_{j}}{b_{j}^{2}} & \text { for } j=1,2, \ldots, k \\
-\sum_{i=1}^{k} \frac{a_{i}}{b_{i}^{2}} & \text { for } j=k+1 \\
0 & \text { for } j=k+2
\end{array} \quad \text { or } \quad \lambda_{j}^{(2)}= \begin{cases}\frac{a_{j}}{b_{j}^{2}} & \text { for } j=1,2, \ldots, k \\
-\sum_{i=1}^{k} \frac{a_{i}}{2 b_{i}^{2}} & \text { for } j=k+1, k+2\end{cases}\right.
$$

depending on whether $c^{2}+\sum_{i=1}^{k} \frac{a_{i}}{b_{i}}=0$ or not, respectively. Equation $\left.\frac{d^{3} p}{d \zeta^{3}}\right|_{\zeta=0}=0$ also implies that $\lambda_{j}^{(3)}=0$ for $1 \leq j \leq k$.

When $|\xi| \rightarrow \infty$ we can write $\Phi(\zeta)=-(B+\zeta A)=-\zeta\left(A+\zeta^{-1} B\right)$ and consider $-\zeta^{-1} B$ to be a small perturbation of $-A$. The eigenvalues $\mu_{j}\left(\zeta^{-1}\right)$ of $A+\zeta^{-1} B$ are related to those of $\Phi(\zeta)$ by $\lambda_{j}(\zeta)=\zeta \mu_{j}\left(\zeta^{-1}\right)$. The characteristic polynomial of $A+\nu B$ is

$$
q(\mu, \nu)=(-1)^{k}\left(\mu^{2}-c^{2}-\sum_{i=1}^{k} \frac{\nu a_{i}}{\mu+\nu b_{i}}\right) \prod_{i=1}^{k}\left(\mu+\nu b_{i}\right)
$$

where $\nu=\zeta^{-1}$. In the neighborhood of $\nu=0$ we have

$$
\mu_{j}(\nu)=\mu_{j}^{(0)}+\nu \mu_{j}^{(1)}+\nu^{2} \mu_{j}^{(2)}+\ldots
$$

for $1 \leq j \leq k+2$. The eigenvalues of $-A$ satisfy $\left.q(\mu, \nu)\right|_{\nu=0}=0$, hence

$$
\mu_{j}^{(0)}= \begin{cases}0 & \text { for } j=1,2, \ldots, k \\ \pm c & \text { for } j=k+1, k+2\end{cases}
$$

Computing $\left.\frac{d q}{d \nu}\right|_{\nu=0}=0$ we find

$$
(-1)^{k}\left(\mu^{(0)}\right)^{k-1}\left(\left(\left(\mu^{(0)}\right)^{2}-c^{2}\right) \sum_{i=1}^{k}\left(\mu^{(1)}+b_{i}\right)+2\left(\mu^{(0)}\right)^{2} \mu^{(1)}-\sum_{i=1}^{k} a_{i}\right)=0
$$


so that

$$
\mu_{j}^{(1)}= \begin{cases}r_{j} & \text { for } j=1,2, \ldots, k \\ \frac{1}{2 c^{2}} \sum_{i=1}^{k} a_{i} & \text { for } j=k+1, k+2\end{cases}
$$

where $r_{j}$ are roots of $\left.\frac{d^{k} q}{d \nu^{k}}\right|_{\nu=0, \mu_{j}^{(0)}=0}=0$. One can show that

$$
\mu_{j}^{(2)}=\mp\left(\frac{1}{2 c^{3}} \sum_{i=1}^{k} a_{i} b_{i}+\frac{3}{8 c^{5}}\left(\sum_{i=1}^{k} a_{i}\right)^{2}\right)
$$

for $j=k+1, k+2$ by solving $\left.\frac{d^{2} q}{d \nu^{2}}\right|_{\nu=0}=0$.

In higher dimensions the analysis is similar but lengthier, remember that $\xi$ should be replaced by $|\xi|$ therein.

\section{Stability}

Well-posedness of the Cauchy problem described in Definition 3.3 does not rule out the possibility of exponential growth of solutions as time approaches infinity unless $\alpha$ is arbitrarily small or negative. The following definition helps to eliminate exponential instabilities.

Definition 5.1. The Cauchy problem for a constant coefficient operator $\mathcal{L}$ is weakly (strongly) stable if it is weakly or strongly well-posed and the solution $U(t)$ satisfies

$$
\|U(t)\|_{L^{2}\left(\mathbb{R}^{d}\right)} \leq C(1+t)^{s}\left\|U_{0}\right\|_{H^{s}\left(\mathbb{R}^{d}\right)}, \quad t \geq 0
$$

with $C>0$ and $s>0(s=0)$.

A necessary and sufficient condition for weak stability is that all eigenvalues $\lambda_{j}(\xi)$ of $\Phi(i \xi)=-(B+i A(\xi))$ satisfy $\Re\left(\lambda_{j}(\xi)\right) \leq 0$. Furthermore, if the Jordan blocks corresponding to the eigenvalues with $\Re\left(\lambda_{j}(\xi)\right)=0$ are trivial, then the problem is strongly stable (cf. Lemma 2.1 in [23]).

Theorem 5.2. Let $d \geq 1, \xi \neq 0, a_{i}<0$ for all $1 \leq i \leq k$ and $g(0) \geq 0$, then the Cauchy problem for a constant coefficient operator $\mathcal{L}$ is strongly stable.

Proof. By Proposition 4.2, all eigenvalues of $\Phi(i \xi)$ satisfy $\Re\left(\lambda_{j}(\xi)\right) \leq 0$. Since the characteristic polynomial in higher dimensions splits as in Eq. (4.1), $\tilde{p}\left(\lambda, \xi_{1}, \ldots, \xi_{d}\right)=$ $p(\lambda,|\xi|) \lambda^{d-1} \prod_{i=1}^{k}\left(\lambda+b_{i}\right)^{d-1}$, the algebraic multiplicity $m$ of the zero eigenvalue is $m=$ $d-1$ when $g(0)>0$ and $m=d$ if $g(0)=0$. In both cases, $m$ does not change as $\xi$ ranges along $\mathbb{S}^{d-1}$ and moreover algebraic multiplicity is equal to the geometric multiplicity. Note that $\lambda(\xi)=0$ solves $\frac{\partial^{m-1}}{\partial \lambda^{m-1}} \tilde{p}\left(\lambda, \xi_{1}, \ldots, \xi_{d}\right)=0$, but $\xi \cdot \nabla_{\xi} \frac{\partial^{m-1}}{\partial \lambda^{m-1}} \tilde{p}\left(\lambda, \xi_{1}, \ldots, \xi_{d}\right) \neq 0$ at $\lambda=0$. Hence $\Phi(i \xi)$ is of principal type at $\lambda=0$ and the Jordan blocks corresponding to zero eigenvalues are all trivial. By Parseval's relation

$$
\begin{aligned}
\|U(t)\|_{L^{2}\left(\mathbb{R}^{d}\right)} & =\left\|e^{t \Phi(i \xi)} \hat{U}_{0}(\xi)\right\|_{L^{2}\left(\mathbb{R}^{d}\right)}=\left\|P^{-1}(\xi) e^{t J} P(\xi) \hat{U}_{0}(\xi)\right\|_{L^{2}\left(\mathbb{R}^{d}\right)} \\
& \leq\left\|P^{-1}(\xi)\right\|\|P(\xi)\|\left\|U_{0}\right\|_{L^{2}\left(\mathbb{R}^{d}\right)},
\end{aligned}
$$

where $J$ is the Jordan matrix. By Theorem 3.5 the Cauchy problem for $\mathcal{L}$ is strongly well-posed and Proposition 4.5 implies that $\Re\left(\lambda_{j}(\xi)\right) \nrightarrow+\infty$ as $|\xi| \rightarrow+\infty$, so 
$\left\|P^{-1}(\xi)\right\|\|P(\xi)\|$ is bounded by a constant $C>0$ and the claim follows.

REMARK 5.1. For $\Phi(i \xi)$ to be of principal type at $\lambda=0$, it is important that the constant algebraic multiplicity is equal to the geometric multiplicity. Consider, for example, case ii) (b) of Proposition 4.4: the geometric multiplicity of the zero eigenvalue is less than its algebraic multiplicity in any dimension $d \geq 1$. Hence $\Phi(i \xi)$ is not of principal type at $\lambda=0$ and the Jordan matrix contains a non-trivial block. The Cauchy problem for $\mathcal{L}$ is only weakly stable in that case.

Métivier and Zumbrun [15] classify the multiple eigenvalues as algebraically regular, geometrically regular and nonregular. Eigenvalues of constant multiplicity are algebraically regular. If in addition they are semi-simple, then they are geometrically regular. Simple roots are geometrically regular by definition. Geometric regularity implies Majda's block structure condition and provides an optimal characterization of this condition.

Energy decay. Consider for a moment the following viscoelastic wave equation

$$
\phi_{t t}-c^{2} \Delta \phi+\int_{0}^{t} K(t-s) \Delta \phi(s) d s=0, \quad x \in \mathbb{R}^{d},
$$

together with the associated standard energy in Fourier space

$$
\hat{E}(\xi, t)=\frac{1}{2}\left|\hat{\phi}_{t}\right|^{2}+\frac{1}{2} c^{2}|\xi|^{2}|\hat{\phi}|^{2}
$$

The following assumptions on the relaxation kernel $K(t)$ are commonly accepted in the literature:

[A2] $K: \mathbb{R}^{+} \rightarrow \mathbb{R}^{+}$is a non-increasing $\mathcal{C}^{1}$ function and $l=c^{2}-\int_{0}^{\infty} K(s) d s>0$, [A3] $K(0)>0$ and $K^{\prime}(t)<0$ for all $t \geq 0$.

Examples of kernels satisfying the above assumptions are $K(0)(1+t)^{-\nu}$, $K(0) e^{-(1+t)^{\nu}}$ with properly chosen $\nu>1$ and $K(0)>0$.

Assumption [A2] has a physical origin: in statics, i.e. when $\sigma(x, t)=\bar{\sigma}(x)$ and $\epsilon(x, t)=\bar{\epsilon}(x)$ Eq. $(2.3)$ reduces to

$$
\bar{\sigma}(x)=\rho\left(c^{2}-\int_{0}^{\infty} K(s) d s\right) \bar{\epsilon}(x)
$$

so [A2] states that in a viscoelastic medium the equilibrium stress modulus is positive (cf. Eq. (75) in Ref. [20] where the equilibrium stress function is considered).

Theorem 5.3. Assume that $K(t)$ satisfies [A2] and [A3], then the energy of the solution to (5.1) decreases in time.

Proof. Multiplying the Fourier transform of (5.1),

$$
\hat{\phi}_{t t}+c^{2}|\xi|^{2} \hat{\phi}-\int_{0}^{t}|\xi|^{2} K(t-s) \hat{\phi}(s) d s=0,
$$

by $\hat{\phi}_{t}^{*}$ and taking the real part we compute

$$
\frac{1}{2} \frac{d}{d t}\left(\left|\hat{\phi}_{t}\right|^{2}+c^{2}|\xi|^{2}|\hat{\phi}|^{2}\right)=|\xi|^{2} \Re\left(\hat{\phi}_{t}^{*} \int_{0}^{t} K(t-s) \hat{\phi}(s) d s\right)=|\xi|^{2} \Re\left(\hat{\phi}_{t}^{*}(K * \hat{\phi})(t)\right)
$$


where we utilized the first of the following convolutions:

$$
\begin{aligned}
& (K * f)(t)=\int_{0}^{t} K(t-s) f(s) d s, \\
& (K \circledast f)(t)=\int_{0}^{t} K(t-s)|f(s)-f(t)|^{2} d s,
\end{aligned}
$$

defined for any real or complex valued function $f(t)$. Using the second definition one can compute

$$
\frac{d}{d t}\left((K \circledast \hat{\phi})(t)-|\hat{\phi}|^{2} \int_{0}^{t} K(s) d s\right)=\left(K^{\prime} \circledast \hat{\phi}\right)(t)-2 \Re\left(\hat{\phi}_{t}^{*}(K * \hat{\phi})(t)\right)-K(t)|\hat{\phi}|^{2}
$$

Hence substituting the previously obtained expression for $\Re\left(\hat{\phi}_{t}^{*}(K * \hat{\phi})(t)\right)$ and using [A2] and [A3] we have

$$
\begin{array}{r}
\frac{1}{2} \frac{d}{d t}\left(\left|\hat{\phi}_{t}\right|^{2}+|\xi|^{2}|\hat{\phi}|^{2}\left(c^{2}-\int_{0}^{t} K(s) d s\right)+|\xi|^{2}(K \circledast \hat{\phi})(t)\right)= \\
|\xi|^{2}\left(\left(K^{\prime} \circledast \hat{\phi}\right)(t)-K(t)|\hat{\phi}|^{2}\right) \leq 0
\end{array}
$$

By introducing the following functional:

$$
0 \leq \hat{\mathcal{E}}(\xi, t)=\frac{1}{2}\left|\hat{\phi}_{t}\right|^{2}+\frac{1}{2}\left(c^{2}-\int_{0}^{t} K(s) d s\right)|\xi|^{2}|\hat{\phi}|^{2}+\frac{1}{2}|\xi|^{2}(K \circledast \hat{\phi})(t)
$$

then, by (5.2), $\hat{\mathcal{E}}(\xi, t)$ is non-increasing and obeys $\hat{\mathcal{E}}(\xi, t) \leq \hat{\mathcal{E}}(\xi, 0)=\hat{E}(\xi, 0)$, and on the other hand $\hat{E}(\xi, t) \leq c^{2} l^{-1} \hat{\mathcal{E}}(\xi, t)$ so the uniform decay of $\hat{\mathcal{E}}$ implies the uniform decay of $\hat{E}$.

In the present manuscript we have dealt with the kernel $K(t)=-\sum_{i=1}^{k} a_{i} e^{-b_{i} t}$ with constant coefficients and in this case (2.5) reduces to (5.1). If we choose $a_{i}<0$ and

$$
c^{2}-\int_{0}^{\infty} K(s) d s=c^{2}+\sum_{i=1}^{k} \frac{a_{i}}{b_{i}}=g(0)>0,
$$

then assumptions [A2], [A3] hold true and Theorem 5.3 shows that the energy of an absorbing medium dissipates over time. Moreover, since $-K^{\prime}(t) / K(t)$ is bounded from below by a positive constant: $-K^{\prime}(t) / K(t)>b_{k}=\max _{i} b_{i}>0$, it is possible to show that the energy decays exponentially [17], [22].

Finally note that the absorption condition, $\mathbf{D}-\mathbf{C}>0$, stated in Ref. [1] is equivalent to $a_{i}<0$. Indeed, by definition $a_{i}<0$ holds whenever $\tau_{\epsilon i}>\tau_{\sigma i}$ (see Remark 4.1). In the mono-dimensional Zener model $\mathbf{C}=\mu$ and $\mathbf{D}=\mu \tau_{\epsilon} / \tau_{\sigma}$, therefore, $\mathbf{D}-\mathbf{C}>0$ reduces $\tau_{\epsilon}>\tau_{\sigma}$. In higher dimensions, $\tau_{\epsilon i}>\tau_{\sigma i}$ for all $1 \leq i \leq k$, ensures that the tensor $\mathbf{D}-\mathbf{C}$ is positive definite. 


\section{REFERENCES}

[1] E. Bécache, A. Ezziani and P. Joly, A mixed finite element approach for viscoelastic wave propagation, Computational Geosciences, 8(3):255-299, 2005. 1, 1, 5

[2] S. Benzoni-Gavage, D. Serre, Multi-dimensional Hyperbolic Partial Differential Equations: FirstOrder Systems and Applications, Oxford Mathematical Monographs (Oxford University Press, 2007). 3.1

[3] K. D. Blazek, C. Stolk and W. W. Symes, A mathematical framework for inverse wave problems in heterogeneous media, Inverse Problems, 29(6):1-37, 2013. 1

[4] J. M. Carcione, D. Kosloff and R. Kosloff, Wave propagation simulation in a linear viscoacoustic medium, Geophys. J. Int., 93(2):393-401, 1988. 2

[5] C. M. Dafermos, An abstract Volterra equation with applications to linear viscoelasticity, J. Diff. Eqs., 7(3):554-569, 1970. 1

[6] F. Gantmacher, The Theory of Matrices, volume 2 (Chelsea, 1959). 4

[7] T. E. Ghoul, M. Khenissi and B. Said-Houari, On the stability of the Bresse system with frictional damping, J. Math. Anal. Appl., 455(2):1870-1898, 2017. 4

[8] T. Kato, Perturbation Theory for Linear Operators (Springer-Verlag, 1976). 4

[9] J. U. Kim, On the local regularity of solutions in linear viscoelasticity of several space dimensions, Trans. Amer. Math. Soc., 346(2):359-398, 1994. 1

[10] J. L. Lions and E. Magenes, Non-Homogeneous Boundary Value Problems and Applications, (Springer-Verlag, 1972). 1

[11] H.-P. Liu, D. L. Anderson, and H. Kanamori, Velocity dispersion due to anelasticity: implications for seismology and mantle composition, Geophys. J. R. Astr. Soc., 47(1):41-58, 1976. 2

[12] A. Majda and S. Osher, Initial-boundary value problems for hyperbolic equations with uniformly characteristic boundary, Comm. Pure Appl. Math., 28(5):607-675, 1975. 1

[13] P. Markowich and M. Renardy, Lax-Wendroff methods for hyperbolic history value problems, SIAM J. Numer. Anal., 21(1):24-51, 1984. 4

[14] G. Métivier, The block structure condition for symmetric hyperbolic systems, Bull. London Math. Soc., 32(6):689-702, 2000. 1, 3.1

[15] G. Métivier and K. Zumbrun, Hyperbolic boundary value problems for symmetric systems with variable multiplicities, J. Diff. Eqs., 211(1):61-134, 2005. 5.1

[16] S. Mukherjee and G. H. Paulino, The elastic-viscoelastic correspondence principle for functionally graded materials, revisited, J. Appl. Mech., 70(3):359-363, 2003. 1

[17] V. Pata, Exponential stability in linear viscoelasticity, Quart. Appl. Math., 64(3):499-513, 2006. 5

[18] P. S. Petrov, A. D. Zakharenko, and M. Y. Trofimov, The wave equation with viscoelastic attenuation and its application in problems of shallow-sea acoustics, Acoust. Phys., 58(6):700-707, 2012. 2

[19] J. Rauch, Hyperbolic Partial Differential Equations and Geometric Optics, Graduate Studies in Mathematics, volume 133 (Amer. Math. Soc., 2012). 3

[20] M. Renardy, W. J. Hrusa, and J. A. Nohel, Mathematical Problems in Viscoelasticity, Pitman Monographs and Surveys in Pure and Applied Mathematics (John Wiley \& Sons, 1987). 2, 5

[21] P. G. Richards, On wave fronts and interfaces in anelastic media, Bull. Seis. Soc. Amer., 74(6):2157-2165, 1984. 1

[22] B. Said-Houari and S. A. Messaoudi, General decay estimates for a Cauchy viscoelastic wave problem, Comm. Pure Appl. Anal., 13(4):1541-1551, 2014. 5

[23] S. Solem, P. Aursand and T. Flåtten Wave dynamics of linear hyperbolic relaxation systems, J. Hyper. Diff. Eqs., 12(4):655-670, 2015. 1, 5

[24] G. Strang, Accurate partial difference methods II. Non-linear problems, Numerische Mathematik, 6:37-46, 1964. 3

[25] G. Strang, On strong hyperbolicity, J. Math. Kyoto Univ., 6(3):397-417, 1967. 3.4

[26] W.-A. Yong, Basic aspects of hyperbolic relaxation systems, in Advances in the Theory of Shock Waves, Progr. Nonlinear Diff. Eqs. Appl., volume 47 (Birkhäuser Boston, Boston, 2001) 3.1 